\title{
Recursos de las facultades de comunicación para la formación en competencias en el marco del Espacio Europeo de Educación Superior
}

\section{Resources for schools of communication for skill-based learning in the European Higher Education Area framework}

\author{
Javier Sierra Sánchez. Universidad Abat Oliba CEU \\ Francisco Cabezuelo Lorenzo. Universidad CEU San Pablo
}

Recibido: 6-I-2010 - Aceptado: 10-IV-2010

Resumen:

Este artículo versa sobre la adaptación de las facultades de comunicación españolas al Espacio Europeo de Educación Superior (EEES), que centra su modelo pedagógico en la formación en competencias del estudiante. El estudio ofrece el análisis, explicación e interpretación de una encuesta realizada a diez decanos. Los resultados muestran al detalle las herramientas y recursos con los que nuestras facultades se enfrentan ante el bien conocido reto de Bolonia. La encuesta cubre cinco facultades públicas y otras cinco privadas. Concluye que nuestras facultades están técnica y tecnológicamente bien preparadas. La digitalización de muchas herramientas y la implementación de nuevas metodologías es ya una realidad en nuestras aulas. A pesar de todo, este artículo considera que deben dejarse abiertas las puertas a nuevas posibilidades técnicas de mejora en el proceso de enseñanza-aprendizaje de las todavía jóvenes Ciencias de la Comunicación.

Palabras clave:

Comunicación, Universidad, Espacio Europeo de Educación Superior, Recursos Técnicos y Audiovisuales.

Abstract:

This article tells about the adaptation of Spanish schools of Communication to the European Higher Education Area (EHEA), which model focuses on the development of student's skills. This paper is based in the explanation of the output from a survey made to ten deans of schools. The results show in detail the tools and resources that our schools have to face the well-known Bolonia process. The survey covers five public and five public schools. Spanish Communication faculties are very well prepared to face this challenge. Digitalization of tools and use of new methodology is currently a reality in our classrooms. However, we must be aware of new proposals due to the permanent need of updating of the always-new Communication Sciences.

Key Words:

Communication, University, European Higher Education Area, Audiovisual and Technical Resources. 


\section{Introducción: hacia la europeización de la universidad española.}

La universidad española vive un proceso de gran trascendencia para el futuro de la institución y del país. Probablemente, sin ser conscientes, estamos inmersos en una de las transformaciones educativas europeas más importante de todos los tiempos. Tras la integración política y monetaria parece haberle llegado su turno a la educación. Desde que en 1987 se impulsara el programa de movilidad Erasmus hasta la Declaración de Bolonia de 1999 y su posterior implantación progresiva en España y resto de países del Espacio Europeo de Educación Superior, entre 2008 y 2010, muchos son los aspectos que se ven afectados por una reforma ${ }^{1}$ que pretende insistir sobre todo en aspectos tan fundamentales como la movilidad e intercambio de alumnos, el aprendizaje basado en competencias, y la estructuración de las titulaciones y las materias en créditos europeos a través del European Credit Transfer System (ECTS).

Este artículo se centra en la tan debatida cuestión de las competencias, en un primer momento, para pasar en una segunda parte a investigar las infraestructuras de las facultades de Ciencias de la Información o de la Comunicación, si disponen de los medios materiales que garanticen una adecuada adquisición de competencias por los alumnos que estudian grados relacionados con la Comunicación Social, ya en el campo de la Comunicación Audiovisual, el Periodismo o la Publicidad y las Relaciones Públicas. De este modo, se realiza una aproximación descriptivo-analítica de una muestra representativa de centros universitarios en España para conocer si están suficientemente dotados de recursos materiales como para asumir el reto educativo del Plan Bolonia, que también pretende una educación universitaria enfocada a dar soluciones al mercado laboral y la sociedad.

\section{La formación en competencias: dotar de herramientas al alumno.}

Del acercamiento entre la universidad y la realidad empresarial surge uno de los aspectos más novedosos en este contexto, el de la formación basada en competencias. Este concepto apuesta por una formación de tipo más práctica y por la resolución de problemas simulando una situación real del mercado laboral. Esta nueva propuesta no supone ni mucho menos abandonar los contenidos y fundamentos teóricos, pero es cierto que en ocasiones los deja en un segundo plano o, al menos, hace más hincapié en su aplicación práctica. Se trata de dotar al alumno de unas competencias que le sean útil para aprender a lo largo de toda su vida.

Para profundizar en el proceso de armonización de la educación universitaria europea (EEES) recomendamos la lectura de las siguientes declaraciones europeas suscritas en los diferentes encuentros de la Universidad de la Sorbona (1998), Bolonia (1999), Lisboa (2000), Praga (2001), Barcelona (2002) Graz (2003), Berlín (2003), Glasgow (2005), Bergen (2005). 
En la Ley Orgánica 5/2002 de las cualificaciones y de la formación profesional se define competencia como "el conjunto de conocimientos y capacidades que permiten el ejercicio de la actividad profesional conforme a las exigencias de la producción y el empleo". Varios han sido los estamentos, organismos, instituciones o autoridades que se han acercado al debate y parado a reflexionar sobre el tema, con diferentes resultados y aproximaciones. La Oficina Internacional del Trabajo (OIT) entiende que el término competencias abarca "los conocimientos, las aptitudes profesionales y el saber hacer que se dominan y aplican en un contexto específico" (2005: 4). Por su parte, el Parlamento Europeo, a la hora de elaborar el 'Marco Europeo para las Cualificaciones para el Aprendizaje Permanente' habla de competencia como la "capacidad demostrada para utilizar conocimientos ${ }^{2}$, destrezas ${ }^{3}$ y habilidades personales, sociales y metodológicas, en situaciones de estudio o de trabajo y en el desarrollo profesional y personal. En este contexto, define las competencias en términos de responsabilidad y autonomía ${ }^{4}$.

El proyecto europeo Tuning, que consideraba que fomentar las competencias era el objetivo de todo programa académico y que éstas eran puntos de referencia para el diseño y la evaluación de los planes de estudio, definía las competencias como una "combinación dinámica de conocimientos, comprensión, capacidades y habilidades". Las competencias son estructuradas por los expertos autores del informe Tuning conforme a una tipología. "Las competencias se entienden como conocer y comprender (conocimiento teórico de un campo académico, la capacidad de conocer y comprender), saber cómo actuar (la aplicación práctica y operativa del conocimiento a ciertas situaciones) saber cómo ser (los valores como parte integrante de la forma de percibir a los otros y vivir en un contexto social)". De este modo, las competencias representan una combinación de atributos (con respecto al conocimiento y sus aplicaciones, aptitudes, destrezas y responsabilidades) que describen el nivel o grado de suficiencia con que una persona es capaz de desempeñarlos. Por tanto, las competencias se pueden evaluar y desarrollar (González y Wagenaar, 2006: 15).

Por su parte, Barraycoa y Lasaga (2009: 34) han realizado una revisión bibliográfica y un análisis comparativo de las definiciones de este concepto. De este estudio, extraen un conjunto de características que podrían resumirse brevemente del siguiente modo:

2 “Conocimiento: resultado de la asimilación de información gracias al aprendizaje , acervo de hechos, principios, teorías y prácticas relacionados con un campo de estudio o de trabajo concreto; en el Marco Europeo de Cualificaciones, los conocimientos se describen como teórico o fácticos". European Parliament (2007: 12)

3 "Destreza: habilidad para aplicar conocimientos y utilizar técnicas a fin de completar tareas y resolver problemas; en el Marco Europeo de Cualificaciones, las destrezas se describen como cognitivas (fundadas en el uso del pensamiento lógico, intuitivo y creativo) y prácticas (fundadas en la destreza manual y en el uso de métodos, materiales e instrumentos)”. European Parliament (2007: 12)

4 Véase el documento de la Eurocámara (2007: 12-13) 
- $\quad$ El concepto de competencia es genérico y engloba los de habilidad y capacidad.

- Entre las competencias se establecen relaciones y asociaciones de tal manera que se complementan, potencian y perfeccionan.

- $\quad$ Algunas competencias presentan dimensiones interpersonales e individuales. Así por ejemplo, la competencia de orientación al logro puede suponer tanto la capacidad de proponerse objetivos grupales como individuales.

- Cualquier persona puede adquirir y mejorar las competencias en mayor o menor medida, si bien se detecta que algunas personas muestran una predisposición hacia determinadas competencias.

- $\quad$ La competencia no es un fin en sí mismo sino un medio para alcanzar objetivos individuales y/u organizativos.

- $\quad$ La definición de cada competencia debe ser objetivable y permitir la incorporación de mecanismos tanto de autoevaluación como de evaluación externa.

Cada universidad plantea un programa formativo, en el que el alumno debe adquirir y desarrollar en unos niveles satisfactorios una serie de competencias que le capacitan para ejercer con éxito la profesión para la que cualifica el grado, en uno o varios de los perfiles para los que se forma. Las competencias en los estudios de Grado en Comunicación, siguiendo la guía del Libro Blanco (2005:192) de Comunicación de Agencia Nacional de Evaluación de la Calidad y la Acreditación (ANECA) se dividen en cuatro subcategorías:

a) Competencias disciplinares (saber).

b) Competencias profesionales (saber hacer).

c) Competencias académicas.

d) Competencias específicas.

Los informes de los expertos de la ANECA $^{5}$ desarrollan en ese mismo informe un mapa de competencias disciplinares, profesionales, académicas y específicas para los tres grados tradicionales en el campo de la Comunicación, creando así listados de competencias para las titulaciones del Grado en Periodismo, Grado

5 En el que trabajaron las personalidades más significativas del área del Periodismo, la Comunicación Audiovisual y la Publicidad y Relaciones Públicas, representando a las universidades públicas y privadas españolas en las que se imparten los mencionados estudios. La universidad coordinadora fue la Universitat Autònoma de Barcelona y el coordinador el Prof. Dr. Marcial Murciano Martínez. 
en Comunicación Audiovisual y finalmente en el Grado en Publicidad y Relaciones Públicas. El resultado del trabajo se plasma cuantitativamente en el siguiente cuadro.

Tabla 1. Competencias bajo el paraguas del EEES.

\begin{tabular}{|c|c|c|c|}
\hline & Periodismo & Publicidad y RR.PP. & Comunicación Audiovisual \\
\hline Competencias disciplinares & 13 & 22 & 14 \\
\hline Competencias profesionales & 15 & 12 & 23 \\
\hline Competencias académicas & 8 & 6 & 7 \\
\hline Otras competencias más específicas & 3 & 9 & 7 \\
\hline
\end{tabular}

Las universidades europeas, y más en concreto las facultades y escuelas de Ciencias de la Información o de la Comunicación españolas, deben estar bien dotadas de recursos humanos y materiales para garantizar la calidad de los programas formativos de los grados de comunicación. En este artículo pretendemos tomar el pulso a las facultades de comunicación españolas con el objetivo de descubrir qué recursos materiales destinan para la adquisición de competencias.

Este mapa de competencias requiere que las universidades dispongan de los medios necesarios para garantizar parámetros de calidad adecuados tal y como demanda el espíritu de la Declaración de Bolonia y su posterior transposición en el ordenamiento jurídico español ${ }^{6}$. Estas competencias cualifican para trabajar en los siguientes campos conforme a un listado de perfiles profesionales ${ }^{7}$ fijados previamente:

Tabla 2. Perfiles en las titulaciones de Comunicación según la ANECA.

\begin{tabular}{|ll|}
\hline Grado & Salidas profesionales \\
\hline Periodismo & 1. Redactor/a de información periodística en cualquier tipo de soporte. \\
& 2. Redactor/a o responsable de prensa o comunicación institucional \\
& 3. Investigador/a, docente y consultor/a de comunicación. \\
4. Gestor/a de portales y editor/a de contenidos
\end{tabular}

Real Decreto 1393/2007, de 29 de octubre, por el que se establece la ordenación de las enseñanzas universitarias oficiales Extraídas del Libro Blanco de ANECA para los títulos de Grado en Comunicación. 


\begin{tabular}{|ll|}
\hline Comunicación & 1. Director/a y realizador/a audiovisual \\
Audiovisual & 2. Productor/a y gestor/a audiovisual \\
& 3. Guionista audiovisual \\
& 4. Diseñador de producción y postproducción visual y sonora \\
\hline Publicidad y Relaciones & 1. Director/a de comunicación, investigador/a y consultor/a estratégico en \\
Públicas. & Publicidad y RR.PP. \\
& $\begin{array}{l}\text { 2. Investigadores/as, Planificadores/as y Compradores de medios. } \\
\text { 3. Creativo/a y diseñador/a } \\
\text { 4. Gestor/a de comunicación corporativa. }\end{array}$ \\
\hline
\end{tabular}

\section{Hipótesis y objetivos de la investigación: la dotación tecnológica}

En este contexto, esta investigación se plantea dos hipótesis de partida. En primer lugar, parte de la idea de que las facultades en las que se imparten estudios de la rama de Ciencias de la Información/Comunicación españolas cuentan con los recursos materiales suficientes y necesarios para afrontar la transformación educativa que supone el coloquialmente denominado 'Plan Bolonia'. Hay una relación evidente entre la dotación de medios de un centro y la facilidad de este para dotar de competencias y habilidades a sus alumnos. Sin recursos no se puede trabajar. En segundo lugar, este estudio considera que las facultades de Ciencias de la Información o de la Comunicación de nuestro país apuestan por el impulso de las nuevas tecnologías en las tareas de enseñanza-aprendizaje por competencias bajo el paraguas del inminente reto del EEES. Este artículo apuesta por el mayor uso posible de los avances tecnológicos en el aula y en el trabajo autónomo del alumno.

\section{Metas y objetivos de estudio}

Esta investigación tiene como objetivo principal analizar los recursos materiales con los que los centros educativos cuentan o destinan para la formación de los futuros graduados en las diferentes titulaciones de las Ciencias de la Comunicación así como los recursos que destinan para facilitar la labor docente del profesorado.

Del mismo modo, este estudio pretende conocer si existe una uniformidad en la denominación lingüística y conceptual de las facultades que forman en las Ciencias de la Información/Comunicación y sus estudios adaptados al EEES. 


\section{Metodología empleada: encuestas a las universidades españolas}

Para la obtención de datos empíricos se ha empleado la técnica de la encuesta, entendida conforme a las pautas de la profesora Lourdes Vinuesa (2005: 177), es decir, como un procedimiento estadístico que permite captar la opinión de una sociedad o de un grupo social para determinar el sentido y la intensidad de las corrientes de opinión mayoritarias.

Para conocer cómo afrontan las facultades de comunicación el reto universitario del Espacio Europeo de Educación Superior (EEES), se elaboró un cuestionario de treinta y una preguntas de tipo cerrado que ha sido enviado por mail a los decanos o responsables de las facultades de comunicación españolas. El estudio sigue en marcha gracias al Grupo de Investigación en la Formación Universitaria en Comunicación (GIFUCOM) de la Universitat Abat Oliba CEU de Barcelona. Este artículo presenta los resultados de un total de diez facultades representativas de España. Es un número significativo para pulsar el estado de la dotación de recursos para la implantación de grados en comunicación dentro del Plan Bolonia.

La mitad de los centros representados corresponden a universidades privadas y la otra mitad a universidades públicas. Las muestras representan pluralmente diferentes territorios de España, desde Madrid a Cataluña, pasando por la Comunidad Valenciana, Castilla y León, Galicia o Aragón. La investigación sigue en marcha y antes de dos años pretende completar el estudio íntegro de las actuales 45 centros académicos oficiales y universitarios españoles dedicados a las Ciencias de las Información o Comunicación.

Tabla 3. Listado de las diez universidades públicas y privadas que sirven como muestra del estudio.

\begin{tabular}{|lccc|}
\hline Universidad & \multicolumn{1}{c}{ Centro } & Ubicación & Titularidad \\
\hline Universidade da Coruña & Ciencias de la Comunicación & La Coruña & Pública \\
\hline Universitat Jaume I & Ciencias Humanas y Sociales & Castellón & Pública \\
\hline $\begin{array}{l}\text { Universidad Carlos III } \\
\text { de Madrid }\end{array}$ & $\begin{array}{c}\text { Humanidades, Comunicación } \\
\text { y Documentación }\end{array}$ & Madrid & Pública \\
\hline $\begin{array}{l}\text { Universidade de Santiago } \\
\text { de Compostela }\end{array}$ & Ciencias de la Comunicación & Santiago & Pública \\
\hline Universitat Pompeu Fabra & Comunicación & Barcelona & Pública \\
\hline Universidad Nebrija & Ciencias de la Comunicación & Madrid & Privada \\
\hline IE University & IE School of Communication & Segovia & Privada \\
\hline Universidad San Jorge & Ciencias de la Comunicación & Zaragoza & Privada \\
\hline
\end{tabular}




\begin{tabular}{|lccc|}
\hline Universidad San Pablo & $\begin{array}{c}\text { Humanidades y Ciencias } \\
\text { de la Comunicación }\end{array}$ & Madrid & Privada \\
\hline Universidad Católica & Ciencias Sociales y & & \\
San Antonio de Murcia & de la Comunicación & Murcia & Privada \\
\hline
\end{tabular}

\section{Resultados, descripción y análisis de los datos.}

En la actualidad, todas las facultades son concientes de que las formas de enseñanza-aprendizaje han evolucionado y superado la tradicional clase magistral de antaño, que aunque sigue siendo válida, no es la única fórmula posible. Poco a poco el profesor ha ido introduciendo las nuevas tecnologías en el aula. De esta forma, muchos centros poseen además de la tradicional pizarra convencional, un cañón LCD, un equipo de sonido o hasta una pizarra digital. Éstos son algunos de los elementos que el profesor que aplique las metodologías propuestas por el EEES utilizará con frecuencia. Además, muchas de ellas, son herramientas indispensables para incorporar documentos audiovisuales en sus clases. En este apartado del artículo, iremos analizando y describiendo las respuestas obtenidas de las preguntas formuladas en el cuestionario.

Tabla 4. Total de alumnos de cada una de las facultades.

\begin{tabular}{|lcc|}
\hline Nombre de la universidad & Nombre de la Facultad & Total alumnos facultad \\
\hline A Coruña & Ciencias de la Comunicación & 175 \\
\hline IE Universidad & IE School of communication & 64 \\
\hline San Jorge & Ciencias de la Comunicación & 601 \\
\hline Católica de San Antonio & Ciencias Sociales y de la Comunicación & 497 \\
\hline Antonio de Nebrija & Ciencias de la Comunicación & 373 \\
\hline Universitat Jaume I Castellón & Ciencias Humanas y Sociales & 4500 \\
\hline Universidad Carlos III de Madrid & Facultad de Humanidades, \\
& Comunicación y Documentación & 1702 \\
\hline Santiago de Compostela & Ciencias de la Comunicación & 671 \\
\hline CEU San Pablo Madrid & Facultad de Humanidades & 1690 \\
& y Ciencias de la Comunicación & \\
\hline Pompeu Fabra & Facultat de Comunicació & 800 \\
\hline
\end{tabular}

$38\left|n^{\circ} 11\right|$ doxa.comunicación 
Podemos apreciar cómo las universidades públicas albergan un mayor número de alumnos en su facultad, en contraposición, las universidades privadas apuestan por cifra menor ofreciendo una formación más personalizada ${ }^{8}$. La universidad privada sigue ofreciendo mayores garantías para una enseñanza más individualizada, personal y asistida.

Una de las transformaciones educativas que promueve el Plan Bolonia es la autonomía del trabajo del alumno y el autoaprendizaje como técnica de adquisición de competencias y conocimiento. De este modo, la biblioteca se convierte en un espacio estratégico y fundamental para el aprendizaje. El alumno debe desarrollar en solitario o en grupo trabajos prácticos, pero también teóricos. A pesar del auge de los recursos digitales, las tradicionales herramientas bibliográficas en papel siguen siendo insustituibles. iQué recursos tienen nuestras bibliotecas universitarias? Además de las de la Jaime I, la Pompeu Fabra y la Carlos III de Madrid parecen contar con los mayores fondos bibliográficos.

La financiación pública de estas instituciones les hace contar con mayores recursos económicos que mayoritariamente repercuten en una mayor cifra de libros y cintas de vídeo o películas en DVD en sus estanterías. La Universitat Pompeu Fabra de Barcelona es la que cuenta con mayor número de películas. Supera los 12.000 títulos de documentales, cortos y largometrajes.

Tabla 5. Fondos biliográficos.

\begin{tabular}{|lcc|}
\hline Nombre de la universidad & Nombre de la Facultad & No libros biblioteca \\
\hline A Coruña & Ciencias de la Comunicación & 10000 \\
\hline IE Universidad & IE school of communication & 16179 \\
\hline San Jorge & Ciencias de la Comunicación & 1880 \\
\hline Católica de San Antonio & Ciencias Sociales y de la Comunicación & 6687 \\
\hline Antonio de Nebrija & Ciencias de la Comunicación & 56100 \\
\hline Universitat Jaume I Castellón & Ciencias Humanas y Sociales & 300000 \\
\hline Universidad Carlos III de Madrid & Facultad de Humanidades, & 67084 \\
& Comunicación y Documentación & \\
\hline Santiago de Compostela & Ciencias de la Comunicación & 29000 \\
\hline
\end{tabular}

8 Los datos de la facultad de Ciencias Humanas y Sociales de la Universitat Jaume I de Castellón (4.500 alumnos) no corresponden exactamente a alumnos que estudian las licenciaturas/grados de comunicación/información, sino que facilitaron el número total de alumnos que estudian en esa facultad contabilizando alumnos de otras propuestas formativas que están en el mismo edificio. 


\begin{tabular}{|lcc|}
\hline CEU San Pablo Madrid & $\begin{array}{c}\text { Facultad de Humanidades y } \\
\text { Ciencias de la Comunicación }\end{array}$ & 15747 \\
\hline Pompeu Fabra & Facultat de Comunicació & 98155 \\
\hline
\end{tabular}

Tabla 6. Fondos de las videotecas.

\begin{tabular}{|lcc|}
\hline Nombre de la universidad & Nombre de la Facultad & No Películas \\
\hline A Coruña & Ciencias de la Comunicación & 2000 \\
\hline IE Universidad & IE school of communication & 982 \\
\hline San Jorge & Ciencias de la Comunicación & 16 \\
\hline Católica de San Antonio & Ciencias Sociales y de la Comunicación & 125 \\
\hline Antonio de Nebrija & Ciencias de la Comunicación & 1648 \\
\hline Universitat Jaume I Castellón & Ciencias Humanas y Sociales & 4000 \\
\hline Universidad Carlos III de Madrid & Facultad de Humanidades, & 7819 \\
& Comunicación y Documentación & 3700 \\
\hline Santiago de Compostela & Ciencias de la Comunicación & 2667 \\
\hline CEU San Pablo Madrid & Facultad de Humanidades y Ciencias de la Comunicación & 12207 \\
\hline Pompeu Fabra & Facultat de Comunicació & \\
\hline
\end{tabular}

En este caso las facultades pertenecientes a universidades públicas ofrecen un mayor número de recursos videográficos y de libros que las universidades privadas. Las nuevas tecnologías están presentes en la vida universitaria. Veamos a continuación la dotación de ordenadores de que disponen las facultades para que puedan ser utilizados por los alumnos.

Tabla 7. Ordenadores de sobremesa.

\begin{tabular}{|llc|}
\hline Nombre de la universidad & Nombre de la Facultad & $\begin{array}{c}\text { No ordenadores } \\
\text { Pc Sobremesa }\end{array}$ \\
\hline A Coruña & Ciencias de la Comunicación & 80 \\
\hline IE Universidad & IE school of communication & 85 \\
\hline San Jorge & Ciencias de la Comunicación & 19 \\
\hline
\end{tabular}




\begin{tabular}{|lcc|}
\hline Católica de San Antonio & Ciencias Sociales y de la Comunicación & 225 \\
\hline Antonio de Nebrija & Ciencias de la Comunicación & 33 \\
\hline Universitat Jaume I Castellón & Ciencias Humanas y Sociales & 300 \\
\hline Universidad Carlos III de Madrid & $\begin{array}{c}\text { Facultad de Humanidades, } \\
\text { Comunicación y Documentación }\end{array}$ & 240 \\
\hline Santiago de Compostela & Ciencias de la Comunicación & 127 \\
\hline CEU San Pablo Madrid & $\begin{array}{c}\text { Facultad de Humanidades y } \\
\text { Ciencias de la Comunicación }\end{array}$ & 180 \\
\hline Pompeu Fabra & Facultat de Comunicació & 475 \\
\hline
\end{tabular}

Tabla 8. Ordenadores portátiles para alumnos.

\begin{tabular}{|lcc|}
\hline Nombre de la universidad & Nombre de la Facultad & No portátiles \\
\hline A Coruña & Ciencias de la Comunicación & 0 \\
\hline IE Universidad & IE school of communication & 0 \\
\hline San Jorge & Ciencias de la Comunicación & 601 \\
\hline Católica de San Antonio & Ciencias Sociales y de la Comunicación & 0 \\
\hline Antonio de Nebrija & Ciencias de la Comunicación & 0 \\
\hline Universitat Jaume I Castellón & Ciencias Humanas y Sociales & 30 \\
\hline Universidad Carlos III de Madrid & Facultad de Humanidades, & 18 \\
& Comunicación y Documentación & 6 \\
\hline Santiago de Compostela & Ciencias de la Comunicación & 4 \\
\hline CEU San Pablo Madrid & Facultad de Humanidades y & \\
\hline Pompeu Fabra & Ciencias de la Comunicación & 40 \\
\hline
\end{tabular}

Las nuevas tecnologías de la información y la comunicación son una pieza fundamental en el desarrollo de competencias académicas y profesionales. Por ello, las universidades no escatiman en ordenadores que faciliten la familiarización de los alumnos con entornos tecnológicos. La mayoría de las facultades han optado 
por la compra de ordenadores de sobremesa. Sin embargo, otras universidades más innovadoras, han preferido optar por la compra de ordenadores portátiles para que los alumnos puedan tener la capacidad de movilidad, es decir, de desarrollar sus trabajos en el lugar que necesiten. En este sentido, destaca la apuesta de la Universidad San Jorge, que dota de un ordenador portátil (pc tablet) a cada uno de sus alumnos.

Veamos la dotación de recursos que poseen las facultades de comunicación para capacitar a los alumnos en las competencias profesionales que tienen que ver con el manejo, realización y producción de materiales sonoros en un campo tan importante como es la Radio y Producción de Sonido.

Tabla 9. Estudios de radio.

\begin{tabular}{|lcc|}
\hline Nombre de la universidad & Nombre de la Facultad & No Estudios de Radio \\
\hline A Coruña & Ciencias de la Comunicación & 1 \\
\hline IE Universidad & IE school of communication & 1 \\
\hline San Jorge & Ciencias de la Comunicación & 3 \\
\hline Católica de San Antonio & Ciencias Sociales y de la Comunicación & 2 \\
\hline Antonio de Nebrija & Ciencias de la Comunicación & 1 \\
\hline Universitat Jaume I Castellón & Ciencias Humanas y Sociales & 5 \\
\hline Universidad Carlos III de Madrid & Facultad de Humanidades, & 4 \\
& Comunicación y Documentación & 3 \\
\hline Santiago de Compostela & Ciencias de la Comunicación & 3 \\
\hline CEU San Pablo Madrid & Facultad de Humanidades y & \\
\hline Pompeu Fabra & Ciencias de la Comunicación & 4 \\
\hline
\end{tabular}

Tabla 10. Estudios de grabación/edición de audio.

\begin{tabular}{|lcc|}
\hline Nombre de la universidad & Nombre de la Facultad & $\begin{array}{c}\text { Estudios de grabación/ } \\
\text { edición audio }\end{array}$ \\
\hline A Coruña & Ciencias de la Comunicación & 3 \\
\hline IE Universidad & IE school of communication & 0 \\
\hline San Jorge & Ciencias de la Comunicación & 3 \\
\hline
\end{tabular}




\begin{tabular}{|lcc|}
\hline Católica de San Antonio & Ciencias Sociales y de la Comunicación & 1 \\
\hline Antonio de Nebrija & Ciencias de la Comunicación & 1 \\
\hline Universitat Jaume I Castellón & Ciencias Humanas y Sociales & 6 \\
\hline Universidad Carlos III de Madrid & $\begin{array}{c}\text { Facultad de Humanidades, } \\
\text { Comunicación y Documentación }\end{array}$ & 0 \\
\hline Santiago de Compostela & Ciencias de la Comunicación & 3 \\
\hline CEU San Pablo Madrid & $\begin{array}{c}\text { Facultad de Humanidades y } \\
\text { Ciencias de la Comunicación }\end{array}$ & 1 \\
\hline Pompeu Fabra & Facultat de Comunicació & 4 \\
\hline
\end{tabular}

Como mínimo, cada facultad posee un estudio de radio. El número de estudios está en proporción al número de alumnos que albergan en la facultad. De este modo, las universidades como IE Universidad que tienen un menor número de alumnos tan sólo ofrecen un estudio de radio. La universidad que ofrece la mejor dotación de recursos de radio (dentro de esta muestra) es la Universitat Jaume I de Castellón.

Veamos cuáles son las propuestas formativas que eligen los centros para formar a sus alumnos en las competencias que tienen que ver con la edición, producción y postproducción de audio. No todos los centros usan la misma tecnología. El software es muy variado de un centro a otro.

Tabla 11. Programas de audio (software).

Porcentaje de utilización de los programas de audio que se emplean en los estudios de Comunicación para la edición, producción y postproducción de audio.

\begin{tabular}{|cc|}
\hline GarageBand & $3 \%$ \\
\hline Avid & $3 \%$ \\
\hline Logic Pro & $3 \%$ \\
\hline Dalet & $3 \%$ \\
\hline Adobe Audition & $7 \%$ \\
\hline Audacity & $20 \%$ \\
\hline WaveLab & $0 \%$ \\
\hline Cool Edit & $3 \%$ \\
\hline
\end{tabular}




\begin{tabular}{|cc|}
\hline Mar4win & $13 \%$ \\
\hline Viva & $7 \%$ \\
\hline X-Frame & $10 \%$ \\
\hline Pro-tools & $28 \%$ \\
\hline
\end{tabular}

No existe un acuerdo homogéneo. Cada facultad pone a disposición del profesor un software diferente. Los dos más extendidos en este sentido son 'Pro-tools' con un 28 por ciento (software de pago) y el programa 'Audacity' con un 20 por ciento (software libre).

Veamos a continuación la dotación de recursos que disponen para la adquisición de competencias que tienen que ver con el registro y manipulación de la imagen estática y en movimiento, algo que es realmente importante para estudios como el Grado en Comunicación Audiovisual.

Tabla 12. Platós de TV.

\begin{tabular}{|lcc|}
\hline Nombre de la universidad & Nombre de la Facultad & No de Platós \\
\hline A Coruña & Ciencias de la Comunicación & 1 \\
\hline IE Universidad & IE School of communication & 1 \\
\hline San Jorge & Ciencias de la Comunicación & 1 \\
\hline Católica de San Antonio & Ciencias Sociales y de la Comunicación & 1 \\
\hline Antonio de Nebrija & Ciencias de la Comunicación & 1 \\
\hline Universitat Jaume I Castellón & Ciencias Humanas y Sociales & 2 \\
\hline Universidad Carlos III de Madrid & Facultad de Humanidades, & 2 \\
\hline Santiago de Compostela & Comunicación y Documentación & 2 \\
\hline CEU San Pablo Madrid & Ciencias de la Comunicación & 2 \\
\hline Pompeu Fabra & Facultad de Humanidades y & 3 \\
\hline
\end{tabular}

Debido a la gran inversión económica que supone la dotación de un plató de televisión, vemos que oscilan de uno a tres platós o estudios de televisión. La mayoría de universidades privadas optan por uno. Destacan de forma sobresaliente la Universitat Pompeu Fabra y la Universidad San Pablo CEU de Madrid, que están realmente bien preparadas en cuanto a instalaciones y materiales audiovisuales se refiere.

44 | n 11 | doxa.comunicación 
Tabla 13. Cámaras de estudio/plató de TV.

\begin{tabular}{|lcc|}
\hline Nombre de la universidad & Nombre de la Facultad & Cámaras de estudio/plató \\
\hline A Coruña & Ciencias de la Comunicación & 0 \\
\hline IE Universidad & IE School of communication & 5 \\
\hline San Jorge & Ciencias de la Comunicación & 3 \\
\hline Católica de San Antonio & Ciencias Sociales y de la Comunicación & 5 \\
\hline Antonio de Nebrija & Ciencias de la Comunicación & 5 \\
\hline Universitat Jaume I Castellón & Ciencias Humanas y Sociales & 5 \\
\hline Universidad Carlos III de Madrid & Facultad de Humanidades, & 5 \\
& Comunicación y Documentación & \\
\hline Santiago de Compostela & Ciencias de la Comunicación & 8 \\
\hline CEU San Pablo Madrid & Facultad de Humanidades y Ciencias de la Comunicación & 8 \\
\hline Pompeu Fabra & Facultat de Comunicació & 10 \\
\hline
\end{tabular}

Hay universidades como la Universidade da Coruña y la Universidad San Jorge que al estar terminando la obras de construcción del plató en el momento de hacer la encuesta no tenían aún compradas las cámaras de estudio. Este tipo de cámaras con conexión triax ${ }^{9}$ tiene un coste más elevado que las cámaras ENG ${ }^{10}$.

Los centros universitarios a la hora de comprar cámaras de estudio con conexión tríax, se inclinan por dos fabricantes de calidad y prestigio como son Sony y Panasonic. De nuevo, en este caso, la marca internacional Sony, de origen japonés, sigue siendo la referencia en el mercado profesional y de la formación en materia de Comunicación Audiovisual.

Tabla 14. Número de cámaras ENGs.

\begin{tabular}{|llc|}
\hline Nombre de la universidad & Nombre de la Facultad & no Cámaras ENG \\
\hline A Coruña & Ciencias de la Comunicación & 15 \\
\hline IE Universidad & IE School of communication & 8 \\
\hline San Jorge & Ciencias de la Comunicación & 8 \\
\hline \hline
\end{tabular}

9 Para profundizar sobre el sistema de conexión tríax puede consultarse el siguiente enlace: http://www.megaservice.com.ar/Productos/telemetrics.htm

10 Estas siglas hacen referencia al nombre completo Electronic News Gathering, coloquialmente denominadas ENGs. 


\begin{tabular}{|lcc|}
\hline Católica de San Antonio & Ciencias Sociales y de la Comunicación & 15 \\
\hline Antonio de Nebrija & Ciencias de la Comunicación & 8 \\
\hline Universitat Jaume I Castellón & Ciencias Humanas y Sociales & 20 \\
\hline \multicolumn{2}{|l|}{ Universidad Carlos III de Madrid } & $\begin{array}{c}\text { Facultad de Humanidades, } \\
\text { Comunicación y Documentación }\end{array}$ \\
\hline Santiago de Compostela & Ciencias de la Comunicación & 21 \\
\hline CEU San Pablo Madrid & Facultad de Humanidades y Ciencias de la Comunicación & 20 \\
\hline Pompeu Fabra & Facultat de Comunicació & 22 \\
\hline
\end{tabular}

En el caso de las cámaras ENGs es mayor el número de cámaras que poseen las facultades debido a la bajada paulatina de precios en los últimos años en el mercado de la tecnología digital en la captación de imágenes. La tecnología digital cada vez es más asequible y está más popularizada.

Cada marca desarrolla su formato de registro y en muchos casos se vuelven incompatibles entre sí. La siguiente tabla muestra los diferentes formatos de las marcas Sony, Panasonic, JVC y Canon.

Tabla 15. Marcas y formatos (Cámaras).

\begin{tabular}{|cc|}
\hline Marca & Formato \\
\hline Sony & DVCam \\
\hline Panasonic & DVC-Pro \\
\hline JVC & DV \\
\hline Canon & DV \\
\hline
\end{tabular}

El mercado se reparte entre cuatro fabricantes siendo la firma asiática Sony la marca de referencia en los equipos de ENG en las facultades y centros universitarios donde se forman los comunicadores e informadores del mañana.

Tabla 16. Estaciones no lineales de vídeo

\begin{tabular}{|llc|}
\hline Nombre de la universidad & Nombre de la Facultad & $\begin{array}{c}\text { No estaciones no } \\
\text { lineales vídeo }\end{array}$ \\
\hline A Coruña & Ciencias de la Comunicación & 80 \\
\hline IE Universidad & IE School of communication & 4 \\
\hline
\end{tabular}




\begin{tabular}{|lcc|}
\hline San Jorge & Ciencias de la Comunicación & 15 \\
\hline Católica de San Antonio & Ciencias Sociales y de la Comunicación & 16 \\
\hline Antonio de Nebrija & Ciencias de la Comunicación & 63 \\
\hline Universitat Jaume I Castellón & Ciencias Humanas y Sociales & 7 \\
\hline Universidad Carlos III de Madrid & $\begin{array}{c}\text { Facultad de Humanidades, } \\
\text { Comunicación y Documentación }\end{array}$ & 10 \\
\hline Santiago de Compostela & Ciencias de la Comunicación & 19 \\
\hline CEU San Pablo Madrid & Facultad de Humanidades y Ciencias de la Comunicación & 24 \\
\hline Pompeu Fabra & Facultat de Comunicació & 12 \\
\hline
\end{tabular}

Tabla 17. Estaciones analógicas de vídeo.

\begin{tabular}{|lcc|}
\hline Nombre de la universidad & Nombre de la Facultad & $\begin{array}{c}\text { No estaciones } \\
\text { analógicas vídeo }\end{array}$ \\
\hline A Coruña & Ciencias de la Comunicación & 0 \\
\hline IE Universidad & IE School of communication & 1 \\
\hline San Jorge & Ciencias de la Comunicación & 0 \\
\hline Católica de San Antonio & Ciencias Sociales y de la Comunicación & 3 \\
\hline Antonio de Nebrija & Ciencias de la Comunicación & 1 \\
\hline Universitat Jaume I Castellón & Ciencias Humanas y Sociales & 2 \\
\hline Universidad Carlos III de Madrid & Facultad de Humanidades, & 2 \\
& Comunicación y Documentación & 2 \\
\hline Santiago de Compostela & Ciencias de la Comunicación & 3 \\
\hline CEU San Pablo Madrid & Facultad de Humanidades y Ciencias de la Comunicación & 0 \\
\hline Pompeu Fabra & Facultat de Comunicació & 3 \\
\hline
\end{tabular}

Los datos expuestos confirman y vuelven a dejar bien claro que la consolidación de la tecnología digital en las facultades de comunicación es toda una realidad. El abaratamiento de costes de la tecnología digital ha provocado que las facultades puedan dotarse de un mayor número de medios y de mejor calidad con los que dotar a sus alumnos de herramientas y competencias para convertirlos en profesionales de éxito. Los datos 
también confirman que el desplome de las estaciones de montaje analógico, que, o bien han descendido, o bien en algunas facultades ni siquiera existen a día de hoy.

Tabla 18. Software para edición, producción y postproducción.

\begin{tabular}{|cc|}
\hline $\begin{array}{l}\text { Porcentaje de utilización de programas destinados a la edición, producción y postproducción de vídeo } \\
\text { que se utilizan en la formación de estudiantes de Ciencias de la Comunicación }\end{array}$ \\
\hline Avid Liquid & $5 \%$ \\
\hline Avid Xpress & $30 \%$ \\
\hline Avid Newscutter & $10 \%$ \\
\hline Avid Composer & $20 \%$ \\
\hline Adobe Premiere & $30 \%$ \\
\hline Sony Vegas & $0 \%$ \\
\hline Final Cut & $5 \%$ \\
\hline
\end{tabular}

Las encuestas también nos demuestran que no existe una uniformidad clara en la propuesta formativa que tiene que ver con la edición, producción y postproducción de vídeo. Las dos marcas que compiten en este aspecto son AVID y Premiere seguidas, pero a gran distancia, por Final Cut.

Pero además del tratamiento de la imagen en movimiento, los alumnos deben conocer y estudiar la imagen fija. La fotografía es una disciplina común a todas las titulaciones de grado en Comunicación. Los laboratorios fotográficos, a pesar del auge de la fotografía digital, siguen existiendo, aunque con finalidades más artísticas y artesanales, que funcionales, tal y como eran en el pasado. La siguiente gráfica ofrece la información cuantitativa sobre la dotación de recursos materiales para la adquisición de competencias que tienen que ver con el registro y manipulación de la imagen estática.

Tabla 19. Número de ampliadoras en laboratorios fotográficos.

\begin{tabular}{|lcc|}
\hline Nombre de la universidad & Nombre de la Facultad & Ampliadoras \\
\hline A Coruña & Ciencias de la Comunicación & 0 \\
\hline IE Universidad & IE School of communication & 15 \\
\hline San Jorge & Ciencias de la Comunicación & 0 \\
\hline Católica de San Antonio & Ciencias Sociales y de la Comunicación & 8 \\
\hline
\end{tabular}




\begin{tabular}{|lcc|}
\hline Antonio de Nebrija & Ciencias de la Comunicación & 8 \\
\hline Universitat Jaume I Castellón & Ciencias Humanas y Sociales & 15 \\
\hline Universidad Carlos III de Madrid & $\begin{array}{c}\text { Facultad de Humanidades, } \\
\text { Comunicación y Documentación }\end{array}$ & 5 \\
\hline Santiago de Compostela & Ciencias de la Comunicación & 8 \\
\hline CEU San Pablo Madrid & $\begin{array}{c}\text { Facultad de Humanidades y } \\
\text { Ciencias de la Comunicación }\end{array}$ & 10 \\
\hline Pompeu Fabra & Facultat de Comunicació & 0 \\
\hline
\end{tabular}

Se observa que aquellas universidades que han apostado desde el inicio por lo digital, descartan los laboratorios fotográficos analógicos en pro de las posibilidades creativas y formativas de la fotografía digital en la que hoy ubicamos las técnicas del fotoperiodismo moderno. De ahí que algunas facultades como las de A Coruña, San Jorge y Pompeu Fabra no contemplen en sus instalaciones un laboratorio fotográfico analógico. Aquellas universidades más veteranas que nacieron en décadas anteriores poseen laboratorio fotográfico analógico y sus correspondientes ampliadoras.

Tabla 20. Número de cámaras digitales-réflex a disposición de los alumnos.

\begin{tabular}{|lcc|}
\hline Nombre de la universidad & Nombre de la Facultad & Cámaras digitales réflex \\
\hline A Coruña & Ciencias de la Comunicación & 8 \\
\hline IE Universidad & IE School of communication & 0 \\
\hline San Jorge & Ciencias de la Comunicación & 5 \\
\hline Católica de San Antonio & Ciencias Sociales y de la Comunicación & 8 \\
\hline Antonio de Nebrija & Ciencias de la Comunicación & 0 \\
\hline Universitat Jaume I Castellón & Ciencias Humanas y Sociales & 10 \\
\hline Universidad Carlos III de Madrid & Facultad de Humanidades, & 15 \\
& Comunicación y Documentación & 0 \\
\hline Santiago de Compostela & Ciencias de la Comunicación & 15 \\
\hline CEU San Pablo Madrid & Facultad de Humanidades y & \\
\hline Pompeu Fabra & Ciencias de la Comunicación & 20 \\
\hline
\end{tabular}


Tabla 21. Número de cámaras analógicas.

\begin{tabular}{|lcc|}
\hline Nombre de la universidad & Nombre de la Facultad & $\begin{array}{c}\text { Cámaras analógicas } \\
\text {-réflex }\end{array}$ \\
\hline A Coruña & Ciencias de la Comunicación & 0 \\
\hline IE Universidad & IE School of communication & 5 \\
\hline San Jorge & Ciencias de la Comunicación & 5 \\
\hline Católica de San Antonio & Ciencias Sociales y de la Comunicación & 0 \\
\hline Antonio de Nebrija & Ciencias de la Comunicación & 5 \\
\hline Universitat Jaume I Castellón & Ciencias Humanas y Sociales & 15 \\
\hline Universidad Carlos III de Madrid & Facultad de Humanidades, & 8 \\
\hline Santiago de Compostela & Comunicación y Documentación & 5 \\
\hline CEU San Pablo Madrid & Ciencias de la Comunicación & 15 \\
\hline Pompeu Fabra & Facultad de Humanidades y Ciencias de la Comunicación & 0 \\
\hline
\end{tabular}

Del análisis de los resultados, vemos como de nuevo, la tendencia es potenciar la tecnología digital. De este modo, vemos cómo las facultades de comunicación de las diferentes universidades, ponen a disposición de los alumnos un mayor número de cámaras digitales que analógicas. Destacamos la dotación de recursos de las universidades Jaume I de Castellón y la Universidad CEU San Pablo de Madrid. Como hemos podido observar, el coste que asumen las facultades de comunicación es muy alto lo que provoca que tengan que contratar personal técnico que salvaguarde los equipos y se encargue del mantenimiento y control de los mismos.

Tabla 22. Número de técnicos audiovisuales por centro.

\begin{tabular}{|lcc|}
\hline Nombre de la universidad & Nombre de la Facultad & $\begin{array}{c}\text { No técnicos } \\
\text { facultad }\end{array}$ \\
\hline A Coruña & Ciencias de la Comunicación & 0 \\
\hline IE Universidad & IE School of communication & 3 \\
\hline San Jorge & Ciencias de la Comunicación & 2 \\
\hline Católica de San Antonio & Ciencias Sociales y de la Comunicación & 2 \\
\hline
\end{tabular}

$50\left|n^{\circ} 11\right|$ doxa.comunicación 


\begin{tabular}{|lcc|}
\hline Antonio de Nebrija & Ciencias de la Comunicación & 2 \\
\hline Universitat Jaume I Castellón & Ciencias Humanas y Sociales & 7 \\
\hline Universidad Carlos III de Madrid & $\begin{array}{c}\text { Facultad de Humanidades, } \\
\text { Comunicación y Documentación }\end{array}$ & 3 \\
\hline Santiago de Compostela & Ciencias de la Comunicación & 3 \\
\hline CEU San Pablo Madrid & Facultad de Humanidades y Ciencias de la Comunicación & 9 \\
\hline Pompeu Fabra & Facultat de Comunicació & 11 \\
\hline
\end{tabular}

Todas las facultades encuestadas, tanto públicas como privadas, a excepción de la Universidade da Coruña, poseen como mínimo dos técnicos para tener cubiertas las necesidades horarias de la actividad académica diaria de clases y prácticas.

Finalmente, en lo que hace referencia a la dotación de recursos tecnológicos y audiovisuales para las tareas docentes en el aula convencional, estos son los resultados obtenidos en la encuesta a las diez universidades seleccionadas, públicas y privadas, de nuestro país.

Tabla 23. Recursos del profesor en el aula convencional.

\begin{tabular}{|c|c|c|c|c|c|c|c|c|}
\hline & 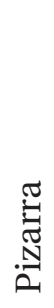 & 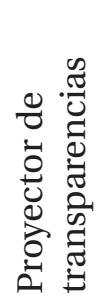 & 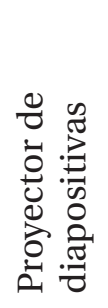 & 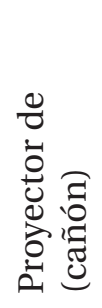 & 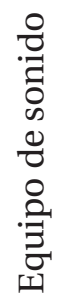 & $\begin{array}{l}2 \\
0 \\
0 \\
0 \\
0 \\
3\end{array}$ & 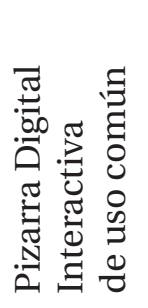 & 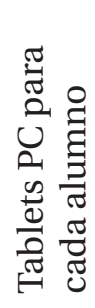 \\
\hline \multicolumn{9}{|l|}{ Nombre de la universidad } \\
\hline Universidade da Coruña & sí & sí & no & sí & sí & sí & no & no \\
\hline IE University & sí & sí & no & sí & sí & sí & no & no \\
\hline U. San Jorge & sí & no & no & sí & sí & no & no & sí \\
\hline U. Católica de San Antonio & sí & sí & no & sí & sí & sí & no & no \\
\hline U. Antonio de Nebrija & sí & sí & sí & sí & sí & sí & no & no \\
\hline U. Jaume I de Castellón & sí & sí & no & sí & sí & sí & no & no \\
\hline
\end{tabular}




\begin{tabular}{|lcccccccc|}
\hline U. Carlos III de Madrid & sí & sí & no & sí & sí & sí & no & no \\
\hline U. Santiago de Compostela & sí & sí & sí & sí & sí & sí & no & no \\
\hline U. San Pablo CEU Madrid & sí & sí & no & sí & sí & sí & no & no \\
\hline U. Pompeu Fabra & sí & sí & sí & sí & sí & sí & sí & no \\
\hline
\end{tabular}

Todas las facultades son concientes de que las formas de enseñanza-aprendizaje han cambiado y que es necesaria la aportación de las nuevas tecnologías en el aula. De esta forma todas poseen: pizarra, cañón LCD, equipo de sonido. Éstos son los elementos básicos que un profesor necesita para incorporar textos audiovisuales en sus clases. Destaca muy positivamente la dotación de recursos para la labor docente del profesor a la Universidad San Jorge (USJ), que apuesta por las 'tablets pc' como herramienta educativa en la formación universitaria. Igualmente, tiene un lugar destacado la Universitat Pompeu Fabra (UPF) que ha introducido la pizarra digital en el aula.

\section{Conclusiones: reflexiones ante el EEES}

Tras el análisis de los resultados obtenidos, pueden establecerse algunas reflexiones a modo de conclusión. El EEES nos exige un cambio de planteamientos. La formación en competencias es uno de los grandes retos de Bolonia. Junto a la adecuada dotación de equipamientos, las facultades necesitarán nuevos espacios para tutorías individuales y grupales, espacio para el trabajo en grupo de los alumnos, menos números por curso y programas formativos más dinámicos y motivadores. Este trabajo pretende hacer una descripción de los equipamientos que tienen las facultades ante el reto de Bolonia, aunque los autores son conscientes y quieren reiterar también una idea clave: la dotación de los equipamientos por si misma no es una variable que permita decir si las facultades están en conjunto bien o mal preparadas para asumir los cambios que establece Bolonia. Hay también otros múltiples factores que juegan un papel igual de importante o incluso más en esa adecuación a Bolonia. Sin duda alguna, más que un reto material, el EEES implica un reto en los planteamientos y formas de interacción entre profesores y alumnos en el cada vez más complejo proceso de enseñanza-aprendizaje.

Respecto a las conclusiones más concretas del estudio, en primer lugar, es preciso recordar que existe una falta de homogenización de la nomenclatura de las facultades de comunicación, en cuanto a su denominación. Debido a que las Ciencias de la Información o de la Comunicación son relativamente jóvenes y también como resultado del pragmatismo de muchas universidades, que ligan unos estudios con otros con el fin de hacerlos más apetecibles a sus alumnos. No hay una denominación común para los centros. Además, en el caso de Es- 
paña, muchos centros son de reciente creación. Igualmente, todavía no hay una gran tradición científica en este campo, si lo comparamos con disciplinas de las Ciencias Exactas y Experimentales. Los estudios de Comunicación, en muchos casos, son estudios que han nacido bajo el auspicio de las facultades de Ciencias Sociales y Jurídicas, y en algunos casos, dentro de las facultades de Humanidades. Sería deseable que hubiese un consenso acerca de la denominación genérica de estos estudios y la necesidad de que estos estudios obtengan su autonomía científica y universitaria. Los cambios acaecidos gracias al Espacio Europeo de Educación Superior son una buena oportunidad para unificar criterios y crear nuevos lazos entre los centros educativos.

Por otro lado, es necesario hacer una reflexión respecto a la dotación de recursos tecnológicos y audiovisuales en nuestros centros. Otra reflexión aparte merece la cuestión de los recursos humanos y docentes. Nuestras facultades cuentan con los recursos bibliográficos y videográficos necesarios para garantizar un apoyo adecuado al alumno en la adquisición de conocimiento.

Existe un aceptable impulso de las tecnologías de la información y de la comunicación en cuanto a la dotación de ordenadores de sobremesa, sin embargo, sería deseable la adquisición de portátiles para el préstamo o cesión a los alumnos para facilitarles la movilidad dentro del recinto educativo universitario. Además, tengamos en cuenta, que del espíritu de Bolonia se desprenden nuevas formas de aprendizaje, y no sólo en el aula. La opción de la tablet pc es un ejemplo válido y aceptable, tal y como se refleja de la experiencia de la Universidad San Jorge, o de las pizarras digitales, en el caso de la Universitat Pompeu Fabra.

Es evidente que nuestras facultades no viven ajenas a la revolución tecnológica a la que estamos asistiendo, por este motivo, están apostando por entornos de trabajo digitales. Están impulsando claramente: la fotografía y el periodismo digital. En este contexto, la radio se convierte en el medio peor dotado, en contraposición de los estudios de televisión.

Respecto a la cuestión del software, es necesario hacer hincapié en una cuestión. Existe una gran dispersión entre las facultades de comunicación a la hora de decidir cuáles son los programas informáticos que mejor capacitarían para la edición de audio y video. Para el retoque fotográfico, sin embargo, hay mayor consenso alrededor de Photoshop.

Concientes del alto coste que asumen las facultades de comunicación en la compra de equipos audiovisuales para la adquisición de competencias profesionales por parte de los alumnos, éstas se dotan como mínimo de dos técnicos para el cuidado, mantenimiento y control de los equipos. Teniendo como mínimo dos técnicos, tienen cubiertas las necesidades horarias de los alumnos para el préstamo y devolución de los equipos en horario de mañana y tarde. 
Todas las facultades españolas estudiadas cuentan con los medios tecnológicos adecuados para facilitar al profesor la tarea docente. En este apartado destacamos de forma sobresaliente a dos facultades por su apuesta por las nuevas tecnologías en las tareas de enseñanza-aprendizaje la Universitat Pompeu Fabra de Barcelona, la Universitat Jaume I de Castellón, entre las públicas, y la Universidad San Pablo CEU de Madrid y Universidad San Jorge de Zaragoza, entre las privadas.

\section{Bibliografía}

Aneca (2005): Libro Blanco. Títulos de grado en Comunicación. Madrid: Agencia Nacional de Evaluación de la Calidad y Acreditación.

Barraycoa, J. y Lasaga, O. (2009): Competencias e inserción laboral: un análisis de la empleabilidad de los recién licenciados en ADE y Económicas. Barcelona: CEU Ediciones.

European Parliament (2007): Posición del Parlamento Europeo adoptada en primera lectura el 24 de octubre de 2007 con vistas a la adopción de la Recomendación 2008/CE del Parlamento Europeo y del Consejo relativa a la creación del Marco Europeo de Cualificaciones para el aprendizaje permanente (EP-PE_TC1-COD (2006) 0163). Luxemburgo: Servicio de Publicaciones del Parlamento Europeo.

González, J. \& Wagenaar, R. (2006): Una introducción a Tuning Educational Structures in Europe. La contribución de las universidades al proceso de Bolonia [en línea], disponible en web: http://tuning.unideusto.org [Consulta: 10.11.2009].

Miguel, M. de (dir.) (2005): Modalidades de enseñanza centradas en el desarrollo de competencias. Orientaciones para promover el cambio metodológico en el Espacio Europeo de Educación Superior. Oviedo: MEC-Universidad de Oviedo.

Organización Internacional del Trabajo (2005): Recomendación sobre el desarrollo de los recursos humanos: educación, formación y aprendizaje permanente. Ginebra: OIT.

Real Decreto 1393/2007, de 29 de octubre, por el que se establece la ordenación de las enseñanzas universitarias oficiales. Boletín Oficial del Estado, 30 de octubre de 2007, núm. 260, p. 44037.

Vinuesa, L. (2005): "La encuesta. Observación extensiva de la realidad social”, en BERGANZA CONDE, M. R. \& RUIZ SAN ROMÁN, J. A. [Coord] (2005). Investigar en Comunicación. Guía práctica de métodos y técnicas de investigación social en Comunicación. Madrid: McGraw Hill, pp. 167-206. 